\title{
Spatial Orientations of Angular Momentum Vectors of Galaxies in Supercluster $S$ [195+027+0022] and Substructure
}

\section{J. R. Malla, W. Saurer and B. Aryal}

Journal of Nepal Physical Society

Volume 7, Issue 2, June 2021

ISSN: 2392-473X (Print), 2738-9537 (Online)

\section{Editors:}

Dr. Binod Adhikari

Dr. Bhawani Joshi

Dr. Manoj Kumar Yadav

Dr. Krishna Rai

Dr. Rajendra Prasad Adhikari

Mr. Kiran Pudasainee

JNPS, 7 (2), 81-88 (2021)

DOI: https://doi.org/10.3126/jnphyssoc.v7i2.38627

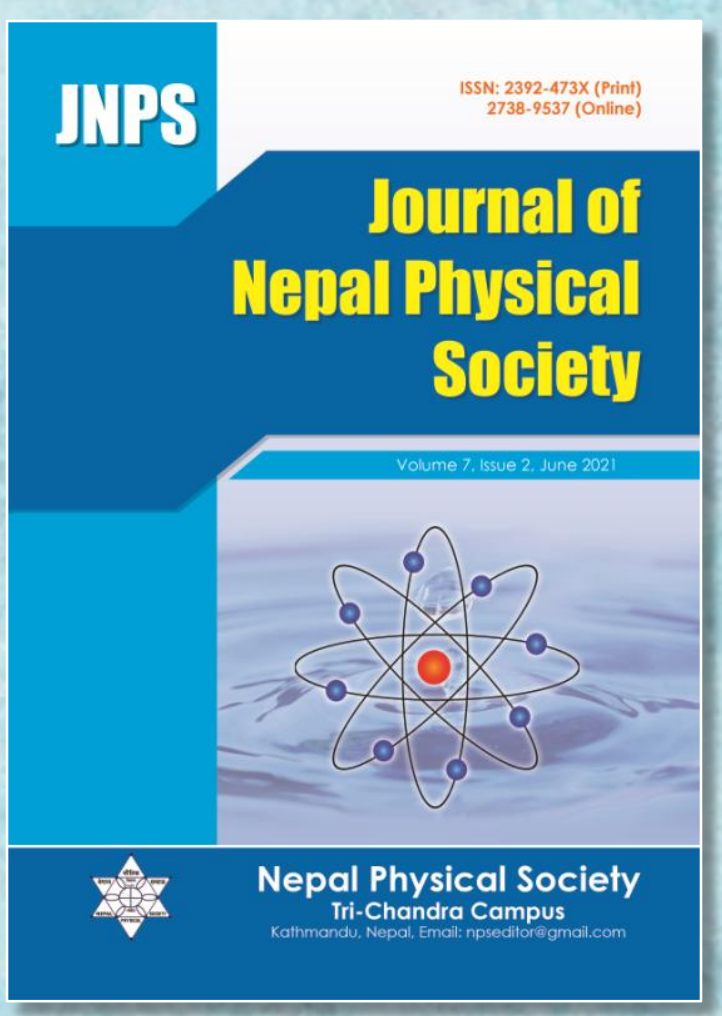

Published by:

Nepal Physical Society

P.O. Box: 2934

Tri-Chandra Campus

Kathmandu, Nepal

Email: nps.editor@gmail.com 


\title{
Spatial Orientations of Angular Momentum Vectors of Galaxies in Supercluster S [195+027+0022] and Substructure
}

\author{
J. R. Malla ${ }^{1, *}$, W. Saurer ${ }^{2}$ and B. Aryal ${ }^{1}$ \\ ${ }^{1}$ Central Department of Physics, Tribhuvan University, Kirtipur, Kathmandu, Nepal \\ ${ }^{2}$ Institute of Astro-particle Physics, Innsbruck University, Austria \\ *Corresponding Email: janak_malla@yahoo.com
}

Received: 15 April, 2021; Revised: 05 May, 2021; Accepted: 27 June, 2021

\begin{abstract}
This paper presents an analysis of the spin vector orientations of SDSS (Sloan Digital Sky Survey) galaxies in the Supercluster S [195+027+0022] using the seventh data release (2008 October). By using the spectroscopic database of galaxies, identified number density map in the region of Superclusters. Several density enhancements are observed, suggesting the possibility of substructure in the Supercluster. Two-dimensional observed parameters that we received from the database are used to compute three-dimensional galaxy rotation axes by applying 'position angle-inclination' method. Apply the selection effects by performing the random simulation method. The expected distribution curves are obtained from the simulation. Chisquare, auto-correlation, and Fourier tests are used to examine non-random effects in the polar and azimuthal angle distributions of the galaxy rotation axes. To check these results with the different galaxy evolution models namely Hierarchy, Primordial, and Pancake model. The result supports the Hierarchy model.
\end{abstract}

Keywords: Galaxy: Evolution, Supercluser, Substructure, Galaxies: cluster

\section{INTRODUCTION}

Galaxy formation is a core theme of cosmology. Galaxies are a slightly laborious topic in astronomy, with many problems relating to their formation and evolution and even with aspects of their structure. Study deeply about the origin and evolution of these large-scale structures. It is necessary to understand how and when galaxies formed and how their constituents have changed with time.

According to Gamow (1952) [1] and Weizsacker (1951) [2]; the observed rotation of the galaxies is very important to understand the origin of the angular momentum of galaxies regarding the beginning of the large scale structures like galaxies and help to get right insight into the initial condition that triggered the formation of these structure Peebles, 1969 [3].

This paper analyzes the spatial orientation of the angular momentum vector of galaxies and their densities in various regions in a Supercluster. In a previous study, a method of searching for sub- clustering in galaxy clusters, based on the analysis of the alignment of the galaxy plane [4]. This work, studied substructure within Supercluster morphologically.

Three main theories advocate predictions concerning the spatial orientation of angular momentum vectors of galaxies. According to the hierarchy model (Peebles, 1969) [3], the angular momentum vector of galaxies is distributed randomly to the plane of the Supercluster. In the turbulence model (Ozernoy, 1978) [5], the angular momentum vectors of galaxies tend to perpendicular to the plane of the Supercluster. The pancake model (Doroshkevich \& Shandarin, 1978) [6] angular momentum vector of galaxy parallel to the cluster plane [7].

\section{MATERIALS AND METHODS}

\section{Data base}

The database used in the current work comprises galaxies located within the survey region of Sloan Digital Sky Survey (SDSS) [8] that have redshift in 
the range of 0.022 to 0.024 . These databases have been obtaining from the SDSS database through our collaborator. There were 2,603 galaxies in the region of interest. Among them, 13 galaxies excluded due to the axial ratio (b/a) is 0.2 , so their inclination angle cannot be determined shown in Fig. 1b. Thus, 2,590 galaxies are in the final sample. The value of right ascension, declination, position angle, and axial ratio (b/a) provided on the database.

\section{Godlowskian Transformation}

The two-dimensional (2-D) information obtained from primary data contains right ascension $(\alpha)$, declination $(\delta)$, position angle $(\mathrm{p})$, and axial ratio (b/a) of galaxies in our Supercluster. Adopted position angle inclination method proposed by Flin and Godlowski to convert two-dimensional given parameters into three-dimensional parameters [5].

The inclination angle (i) of a galaxy is the angle between the normal to the galaxy plane and the observer's line-of-sight. It can be calculated from the Holmberg formula [9],

$$
\cos ^{2} i=\frac{\left(\frac{b}{a}\right)^{2}-q^{*^{2}}}{1-q^{* 2}} \ldots(1)
$$

Here $\mathrm{q}^{*}$ represents the intrinsic flatness factor of the galaxy. The flatness of a disk galaxy depends on the morphological type. Holmberg [9] suggested a value of $\mathrm{q}^{*}=0.20$ for oblate spheroid.

The distribution of the galactic plane and its PA are considered in the equatorial coordinate system. For each galaxy, two angles are determined: the polar angle $(\theta)$, the angle between the normal to the galaxy and the equatorial plane, and azimuthal angle ( $\phi)$, the angle between the projection of this normal on the equatorial plane and directed towards the equatorial center. These angles are given by the equation Flin and Godlowski [10].

$$
\begin{gathered}
\sin \theta=-\operatorname{cosisin} \alpha \pm \sin i \sin p \cos \delta \ldots \text { (2) } \\
\sin \varphi=(\cos \theta)^{-1}[-\cos i \cos \delta \sin \alpha+\sin i(\mp \sin p \sin \delta \sin \alpha \mp \cos p \cos \alpha)] \ldots \text { (3) } \\
\cos \phi=(\cos \theta)^{-1}[-\cos i \cos \delta \cos \alpha+\sin i(\mp \sin p \sin \delta \cos \alpha \mp \cos p \sin \alpha)] \ldots \text { (4) }
\end{gathered}
$$

Here, $\mathrm{i}, \delta, \alpha$, and $\mathrm{P}$ represent the inclination angle, declination, right ascension, and position angles respectively and $\mp$ and \pm indicates the two possible solutions.

\section{METHOD OF ANALYSIS}

The selection effect on the parameters (e.g., position, position angles, inclination angles, etc.) in the right-hand side of equation (2, 3, and 4) affects the distributions of $\theta$ and $\phi$. In the database, three kinds of selection effects can be noticed:

1. Inhomogeneity in the distribution of the position of galaxies

2. Lack of knowledge of position angles (PAs) of face-on galaxies and

3. Lack of edge-on galaxies.

In the selection of position and inclination angle make by the SDSS survey, there may cause various errors in the results. To remove these selection effects, we have to use the numerical simulation method proposed by Aryal and Saurer [11]. For the simulation, the correct spatial distribution of the galaxy rotation axis is supposed to be isotropic. Then, latitude $(\delta)$, longitude $(\alpha)$, inclination angle (i), and position angle (p) can be distributed ran- domly, and equation (2, 3, and 4) used to simulate the corresponding distribution of $\theta$ and $\phi$.

\section{RESULTS AND DISCUSSION}

From the all-sky distribution of galaxies in a largescale structure, the distribution of galaxies is not uniform (As shown in Fig. 1a). There seen some regions are high-density contrast, so must study such density contrast to understand how the overall structure and contrast region affect the evolution and formation of galaxies in that region. In order to identify substructure within the Supercluster S [184+003+00077], try to find the number of galaxies around each galaxy within the different radius values such as $0.5^{\circ}, 0.75^{\circ}, 1.0^{\circ}, 1.25^{\circ}$, and $1.5^{\circ}$. By analyzing the distribution of galaxy in the contour map in various radius values, we choose the particular value of radius for which a clear substructure is seen. In Fig. 2a, we can see four clear high-density contrast regions at $28^{\circ}$ declination. Now, the nearest neighbour distance is increased to $0.75^{\circ}, 1.0^{\circ}$ and $1.25^{\circ}$ from each galaxy. The number density contour map is shown in Figs. 2b, c. The size of the largest substructure at $28^{\circ}$ declination continuously increased. But other different substructure, there is no prominent structure because 
the density contrast region begins to overlap. Due to this region, it is very difficult to separate different substructure to study precisely. Similarly, in Fig. 2d and e, two additional substructures appeared at $20^{\circ}$ declination. When the radius is increased to $1.5^{\circ}$, the lower substructure gets merged. It is, therefore, we set the nearest neighbour radius $\mathrm{r}=1.25^{\circ}$ for the substructure classification.
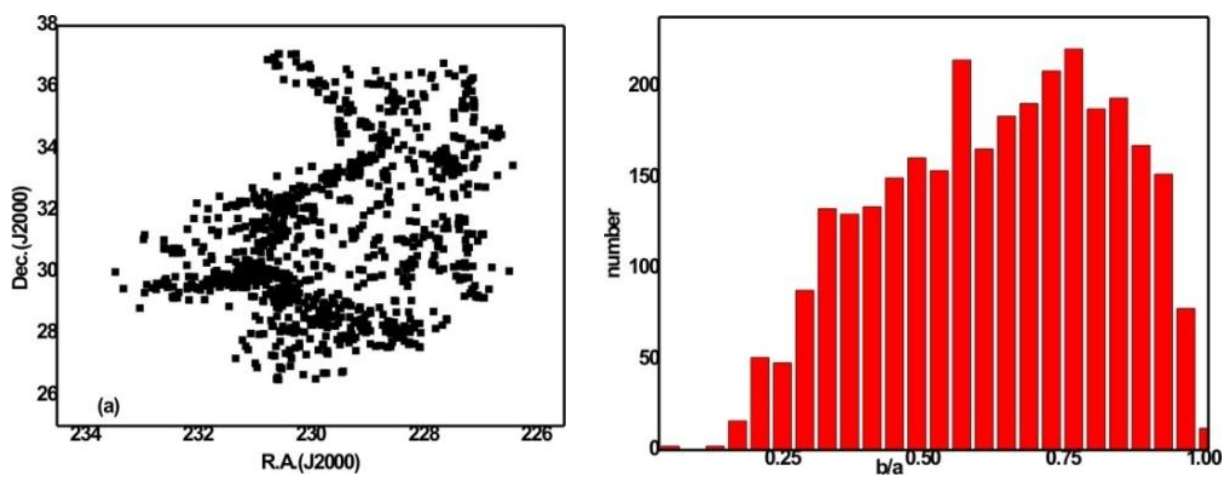

Fig. 1: (a) All-sky distribution of total galaxies of Supercluster S [195+027+0022] in the equatorial coordinate system. R.A. and Dec. represent the right ascension and declination in degree. Each solid circle represents a galaxy. There are 2590 galaxies in this Supercluster. Inhomogeneous distribution of galaxies can be seen. (b) Axial ratio (b/a) distribution of galaxies. The Y-axis represents the number of observed galaxies in the Supercluster S[195+027+0022].

For the substructure identification, "Origin 8.0" and "Matlab 7.0.1" software are used. The contour plot is the process of figuring out the different structures within the given large structure of Supercluster. The contour plot of Supercluster S [195+027+0022] within the different radii of the circle is shown in figure 2 below.
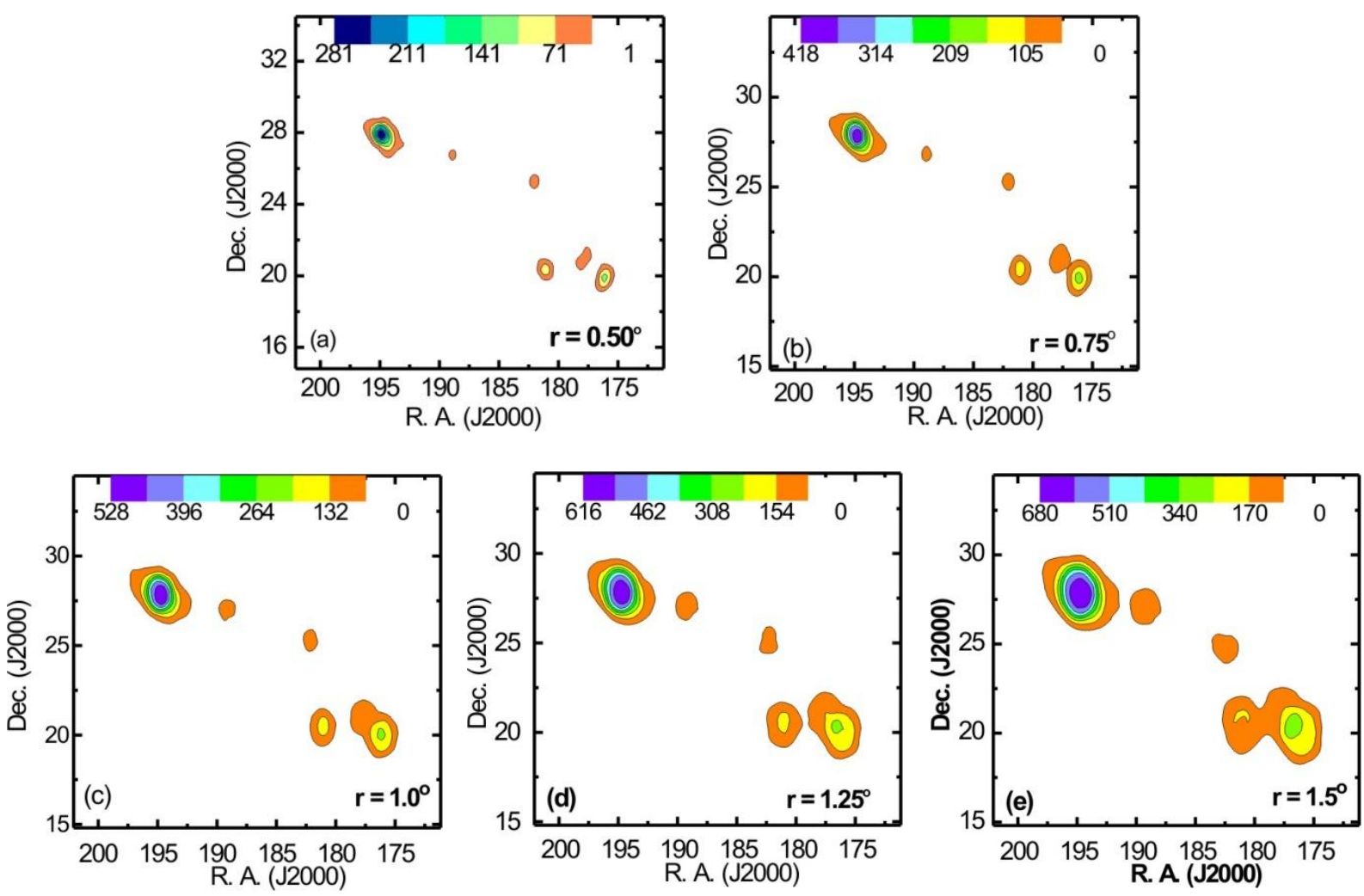

Fig. 2: Number density map of Supercluster $S$ [195+027+0022]. The above diagram, a, b, c, d, and e represent the contour map along the different radii of the circle namely, $0.5^{\circ}, 0.75^{\circ}, 1.0^{\circ}, 1.25^{\circ}$, and $1.50^{\circ}$ respectively. The $\mathrm{X}$-axis and $\mathrm{Y}$-axis represent right ascension (R. A.) and declination (Dec.), respectively. 
This work explained the spatial orientation of the spin vectors of galaxies to the equatorial coordinate system based on the distribution of polar $(\theta)$ and azimuthal $(\phi)$ angle of galaxy rotation axes in Supercluster S [195+027+0022]. By using statistical tools chi-square probability $\mathrm{P}$ $\left(>\chi^{2}\right)$, auto-correlation coefficient $(\mathrm{C} / \mathrm{C}(\sigma))$, first- order Fourier coefficient $\left(\Delta_{11} / \sigma\left(\Delta_{11}\right)\right)$, and Fourier probability $\mathrm{P}\left(>\Delta_{1}\right)$ to test any deviation from expected isotropic distribution. For anisotropy, the limit of chi-square probability $\mathrm{P}$ $\left(>\chi^{2}\right)$ is $<0.050$, auto correlation coefficient $(\mathrm{C} / \mathrm{C}$ $(\sigma))$ is $>1.0$, first order Fourier coefficient $\left(\Delta_{11} / \sigma\right.$ $\left.\left(\Delta_{11}\right)\right)$ is $>1.5$ and Fourier probability $\mathrm{P}\left(>\Delta_{1}\right)$ is < 0.150 respectively. These statistical limits were proposed by Godlowski [12, 13] in a galaxy orientation study.

The statistics for the polar angle $(\theta)$ and azimuthal angle $(\phi)$ distribution is given in Table 1 . In this study of polar angle $(\theta)$, a positive value of first order Fourier coefficient suggests that the spin vectors of galaxies tend to be oriented parallel with respect to the equatorial co-ordinate system. While negative value suggests that perpendicular orientation with respect to equatorial co-ordinate system.

Similarly in the statistics of azimuthal angle $(\phi)$, a positive value of first order Fourier coefficient suggests that the spin vector projections of galaxies tend to point radially outwards the center of the equatorial co-ordinate system while negative value implies spin vectors projection of galaxies tend to orient tangentially with respect to the equatorial coordinate system. In addition to the statistical tests, we also studied the 'humps' (bins with more solutions than expected) and 'dips' (bins with less solutions than expected) in the polar $(\theta)$ and azimuthal angle $(\phi)$ distributions.

In the following Table 1, the first column lists the sample, the second column represents chi-square probability $\left(\mathrm{P}\left(>\chi^{2}\right)\right.$, the third column represents first order Fourier coefficient $\left(\Delta_{11} / \sigma\left(\Delta_{11}\right)\right)$, and the last two columns give first order Fourier probability $\mathrm{P}\left(>\Delta_{1}\right)$ and auto-correlation coefficient $(\mathrm{C} / \mathrm{C}(\sigma))$.

\begin{tabular}{lcccc}
\hline \multicolumn{1}{c}{ Sample } & $\mathbf{P}\left(\boldsymbol{\chi}^{\mathbf{2}}\right)$ & $\left(\boldsymbol{\Delta}_{\mathbf{1 1}} / \boldsymbol{\sigma}\left(\boldsymbol{\Delta}_{\mathbf{1 1}}\right)\right)$ & $\mathbf{P}\left(>\boldsymbol{\Delta}_{\mathbf{1}}\right)$ & $\mathbf{( C / C}(\boldsymbol{\sigma})) \cdot$ \\
\hline Polar Angle & & & & \\
S [195+027+0022] & 0.932 & 0.24 & 0.942 & 0.06 \\
S1[195+028+0022] & 0.924 & 0.57 & 0.848 & -0.29 \\
S2[176+020+0023] & 0.534 & 0.07 & 0.917 & -0.35 \\
S3[181+020+0024] & 0.976 & -0.37 & 0.923 & -0.08 \\
Azimuthal angle & & & & \\
S [195+027+0022] & 0.661 & -0.15 & -0.943 & -0.81 \\
S1[195+028+0022] & 0.416 & -0.27 & 0.999 & -1.08 \\
S2[176+020+0023] & 0.274 & -0.49 & 0.999 & -0.17 \\
S3[181+020+0024] & 0.980 & -2.60 & 0.021 & -0.35 \\
\hline
\end{tabular}

Fig. 2 shows polar $(\theta)$ and azimuthal $(\phi)$ angle distributions of galaxies in the Spercluster $S$ [195+027+0022]. Table 1 shows the statistics for the polar angle $(\theta$-distribution in the Supercluster $S$ [195+027+0022]. In the polar angle distribution, chi-square probability $\left(\mathrm{P}\left(>\chi^{2}\right)\right.$ is found to be 0.932 i.e., $93.2 \%$ (greater than the significant level 0.050 i.e. $5.0 \%$ ). The first order Fourier coefficient $\left(\Delta_{11} / \sigma\left(\Delta_{11}\right)\right)$ is 0.24 (less than significant level i.e., $1.5 \sigma)$, first order Fourier probability $\left(\mathrm{P}\left(>\Delta_{1}\right)\right)$ to be 0.942 i.e., $94.2 \%$ (greater than 0.15 i.e., $15 \%$ ), and auto-correlation coefficient $(\mathrm{C} / \mathrm{C}(\sigma))$ is 0.06 which is less than $1 \sigma$. Thus, all statistical tests suggest isotropic distributions.

Here, the positive value of first order Fourier coefficient suggests that the spin vectors of galaxies tend to be oriented parallel with respect to the equatorial co-ordinate system.

The polar angle $(\theta)$ distribution in the Supercluster $\mathrm{S}$ [195+027+0022] as shown in Fig. 2a, there are no significant hump and dip observed. This is due to binning effect. 

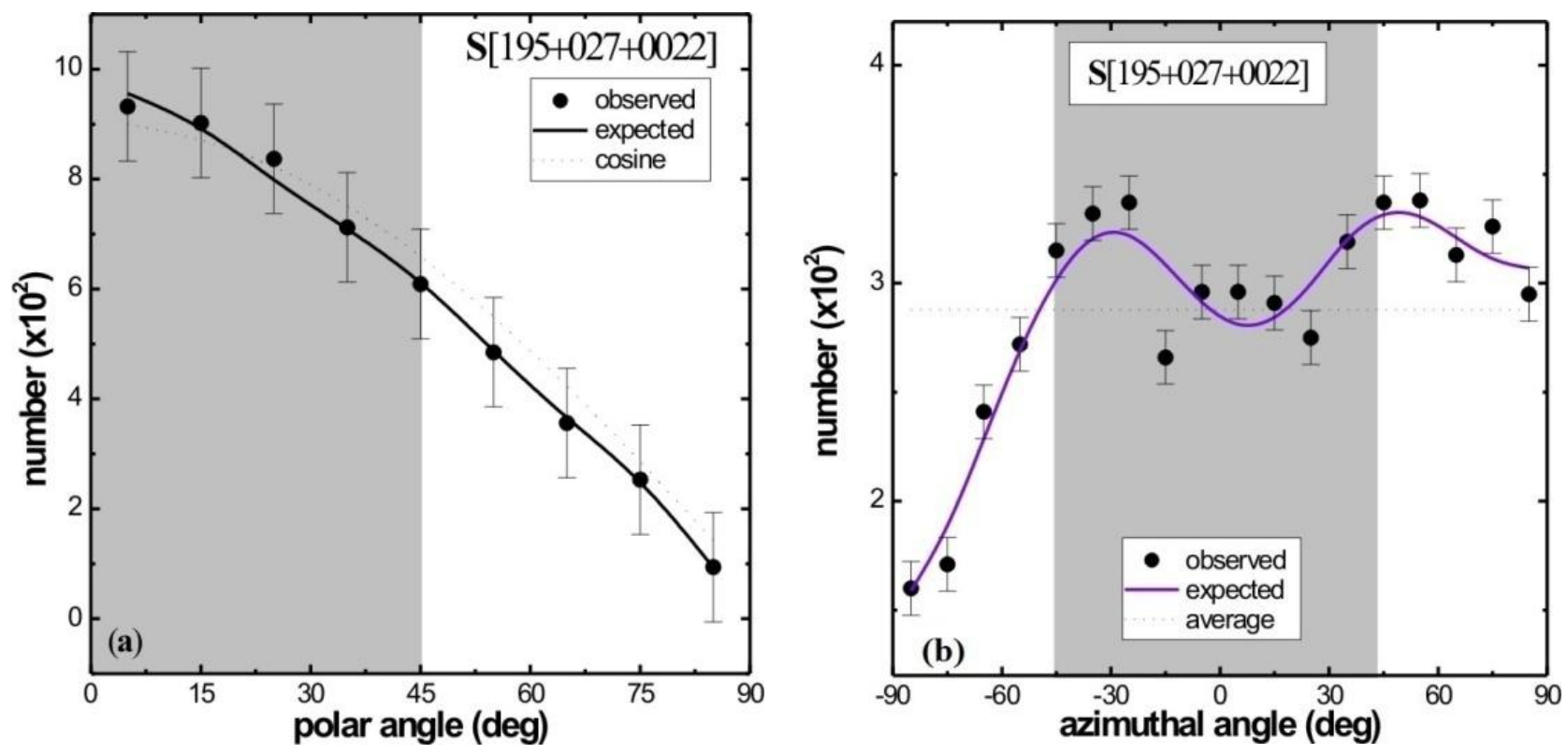

Fig. 2: Polar (a) and azimuthal (b) angle distributions of galaxies in the Supercluster S [195+027+0022]. The solid circles with statistical error bars $( \pm 1 \sigma)$ represent the observed distribution. The solid curves are obtained from random simulation assuming isotropic distributions. The grey shaded region shows a predominance of the Pancake model

(Doroshkevich et al., 1978) [6] if observed solution is found to be more than that of expected.

Fig 2(b) shows the azimuthal angle ( $\phi$ distribution. Table 1 shows the statistics for the azimuthal angle ( $\phi$-distribution in the Supercluster $S$ [195+027+0022]. In the azimuthal angle distribution, chi-square probability $\left(\mathrm{P}\left(>\chi^{2}\right)\right.$ to be 0.661 i.e., $66.1 \%$ which is greater than 0.050 i.e. $5 \%$, first order Fourier coefficient $\left(\Delta_{11} / \sigma\left(\Delta_{11}\right)\right)$ found to be -0.15 less than $1.5 \sigma$, first order Fourier probability $\left(\mathrm{P}\left(>\Delta_{1}\right)\right)$ to be 0.943 i.e., $94.3 \%$ more than 0.150 i.e. $15 \%$ and auto correlation coefficient $(\mathrm{C} / \mathrm{C}(\sigma))$ is -0.81 less than $1 \sigma$. Thus, all statistics tests supporting isotropy.

Here, the negative value of first order Fourier coefficient implies spin vectors projection of galaxies tends to orient tangentially with respect to the equatorial co-ordinate system.

Fig $2 \mathrm{~b}$ shows the azimuthal angle $(\phi)$ distribution, there are no significant hump and dip are seen in the region except, for the region bimodal region ($45^{\circ}<\phi<+45^{\circ}$ ), two dips at $-25^{\circ}$ and $+25^{\circ}$ are observed. This is due to local effect. This suggests that the projection of spin vectors of galaxies tends to orient parallel with respect to the equatorial coordinate system.

Thus, these 'humps' and 'dips' suggest the possibility of grouping or substructuring because of the tidal or gravitational shearing effects between the co-moving galaxies. Thus, the random orientations of the angular momentum vectors of galaxies are found, suggesting the Hierarchical model of the structure formation as suggested by Peebles [3].

Table 1 shows statistics for the polar angle distribution in the substructures S1 [195+028+0022]. In the polar angle $(\theta)$ distribution, chi-square probability $\left(\mathrm{P}\left(>\chi^{2}\right)\right.$ is 0.924 i.e., $92.4 \%$ much more than 0.05 i.e., $5 \%$ significance level, First order Fourier coefficient $\left(\Delta_{11} / \sigma\left(\Delta_{11}\right)\right)$ is found to be 0.57 which is less than $1.5 \sigma$. The First order Fourier probability $\left(\mathrm{P}\left(>\Delta_{1}\right)\right)$ to be 0.848 i.e., $84.8 \% \%$ greater than 0.15 i.e., $15 \%$ significance level and auto-correlation $(\mathrm{C} / \mathrm{C}(\sigma))$ to be found 0.29 less than $1 \sigma$. However, all these statistical tests suggest isotropy. Thus, there is no preferred alignment of galaxies in the Supercluster S1 [195 $+027+0022]$.

The polar angle $(\theta)$ distribution is shown in Fig. 3a below. There are no significant 'humps' and 'dips' are observed, due to binning effect. No preferred alignment i.e. the random orientation is observed supporting the Hierarchical model (strong isotropy) as suggested by Peebles [3].

Table 1 shows statistics for the azimuthal angle distribution in the substructures $\mathrm{S} 1$ [195+028+0022]. In the azimuthal angle $(\phi)$ distribution, chi-square probability $\left(\mathrm{P}\left(>\chi^{2}\right)\right.$ is 0.416 i.e., $41.6 \%$ is greater than 0.005 i.e., $5 \%$. 

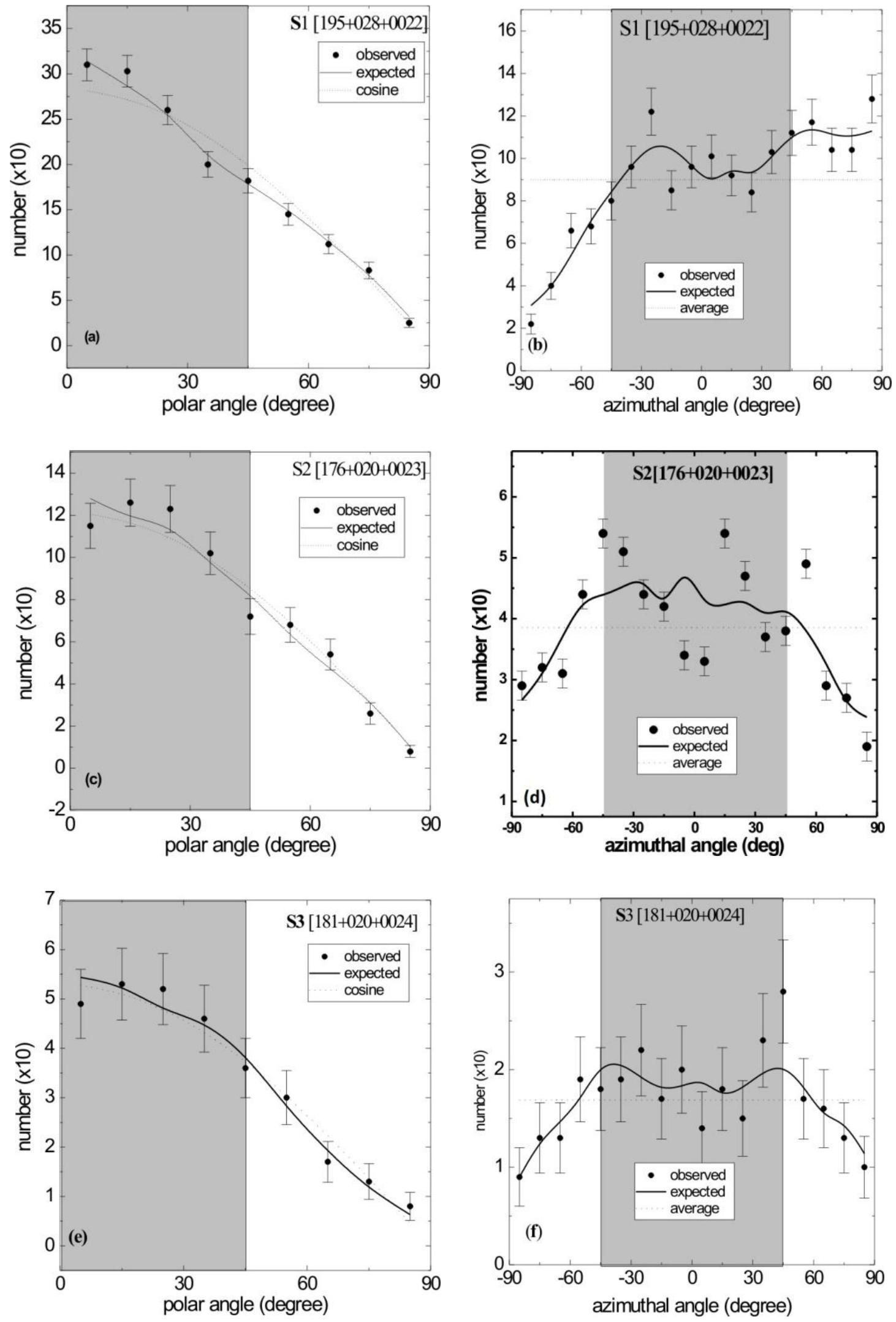

Fig. 3: Polar ( $a, c, e)$ and azimuthal angle (b, d, f) distributions of galaxies in the substructures of Supercluster $\mathrm{S}[195+027+0022]$. Solid circles with statistical error bars $( \pm 1 \sigma)$ represent observed distributions. The expected distributions are represented by solid curves. These solid curves are obtained by performing random simulations. The grey-shaded region supports the pancake model if there is excess solution than expected. 
First order Fourier coefficient $\left(\Delta_{11} / \sigma\left(\Delta_{11}\right)\right)$ is -0.27 which is less than $1.5 \sigma$ level, Fourier probability (P $\left(>\Delta_{1}\right)$ ) is 0.999 i.e., $99.9 \%$ greater than 0.15 i.e., $15 \%$ significant limit whereas value of autocorrelation coefficient $(\mathrm{C} / \mathrm{C}(\sigma))$ is -1.08 less than $1.5 \sigma$. All these statistics support the strong isotropy in $\phi$ distributions of this sample.

The azimuthal angle $(\phi)$ distribution is shown in Fig. $3 \mathrm{~b}$ below. There are three 'humps' at $-65^{\circ}$, $25^{\circ},-85^{\circ}$ and three 'dips' at $-85^{\circ},-15^{\circ}$, and $25^{\circ}$ are seen due to the expected and observed values. Like polar angle, no preferred alignment is seen in the azimuthal angle distribution.

Table 1 shows statistics for the polar angle distribution in the substructures S2 [176+020+0023]. In the polar angle $(\theta)$ distribution, chi-square probability $\left(\mathrm{P}\left(>\chi^{2}\right)\right.$ is found to be 0.533 i.e., $53.3 \%$ greater than 0.05 i.e., $5 \%$ significance level, First order Fourier coefficient $\left(\Delta_{11} / \sigma\left(\Delta_{11}\right)\right)$ is 0.074 less than $1.5 \sigma$. The First order Fourier probability $\left(\mathrm{P}\left(>\Delta_{1}\right)\right)$ to be 0.916 i.e., $91.6 \% \%$ greater than 0.15 i.e., $15 \%$ significance level and auto-correlation $(\mathrm{C} / \mathrm{C}(\sigma))$ to be -0.347 less than $1 \sigma$. Thus, all these statistical tests advocate isotropy.

The polar angle $(\theta)$ distribution is shown in Fig. 3c below. The observed solution for the region $\theta<45^{\circ}$, two 'dips' can be observed within the shaded region at $5^{\circ}$ and bimodal regions (at $45^{\circ}$ ). Then a number of observed (538) and the expected (542) solutions for small angle (up to $45^{\circ}$ ), which indicates 4 less solutions are observed than expected. Also, one hump is seen at $25^{\circ}$ within a limit of $1 \sigma$. There are no humps and dips observed in regions $45^{\circ}<\theta>90^{\circ}$. This is due to binning effects.

The statistics for the azimuthal angle $(\phi)$ distribution in the substructures S2[176+020+0023] as shown in Table 1 . In the azimuthal angle $(\phi)$ distribution, chi-square probability $\left(\mathrm{P}\left(>\chi^{2}\right)\right.$ is 0.273 i.e., $27.3 \%$ more than 0.05 i.e., $5 \%$ significance level, First order Fourier coefficient $\left(\Delta_{11} / \sigma\left(\Delta_{11}\right)\right)$ to be -0.490 which less than $1.5 \sigma$. The First order Fourier probability $\left(\mathrm{P}\left(>\Delta_{1}\right)\right)$ is found to be 0.878 i.e., $87.8 \%$ greater than 0.15 i.e., $15 \%$ significance level, and auto-correlation $(\mathrm{C} / \mathrm{C}(\sigma))$ to be -0.169 less than $1 \sigma$. Here, all these statistical tests supporting isotropy.

In Fig $3 d$, the azimuthal angle $(\phi))$ distribution is shown. In the azimuthal angle $(\phi))$ distribution, at $\left(\phi<45^{\circ}\right)$, small 'dip' is seen at $-65^{\circ}$ and in the bimodal region $\left(-45^{\circ}<\phi<45^{\circ}\right)$ two 'humps' at $-45^{\circ}$ and $15^{\circ}$ are seen and two strong 'dips' at $-5^{\circ}$ and $5^{\circ}$ are seen. The 'humps' and 'dips' in $\phi$-distribution are not so easy to interpret as compared to the $\theta$ distribution. It is because the range of $\phi$ is $-90^{\circ}$ to $90^{\circ}$. At $\left(\phi>45^{\circ}\right)$, one 'hump' at $55^{\circ}$ and a small 'dip' in $85^{\circ}$ can be observed.

Table 1 shows statistics for the polar angle distribution in the substructures S3 $[181+020+0024]$. In the polar angle $(\theta)$ distribution, chi-square probability $\left(\mathrm{P}\left(>\chi^{2}\right)\right.$ is 0.976 greater than 0.05 significance level, First order Fourier coefficient $\left(\Delta_{11} / \sigma\left(\Delta_{11}\right)\right)$ is -0.370 less than $1.5 \sigma$. The First order Fourier probability $\left(\mathrm{P}\left(>\Delta_{1}\right)\right)$ to be 0.923 greater than the 0.15 significance level, and auto-correlation $(\mathrm{C} / \mathrm{C}(\sigma))$ to be -0.082 less than $1 \sigma$. All these statistical tests suggest the isotropic distribution of polar angle, suggesting random orientation of angular momentum of galaxies as suggested by Peebles [3].

The polar angle $\theta$ - distribution is shown in Fig. $3 \mathrm{e}$ below. In polar angle ( $\theta$ distribution, there are no significant 'humps' and 'dips' are seen. This is due to binning effect. Thus, the random orientation of the angular momentum of the galaxies is found, suggesting the hierarchical model of structure formation as suggested by Peebles [3].

The statistics for the azimuthal angle $(\phi)$ distribution in the substructures S3[181+020+0024] as shown in Table 1 . In the azimuthal angle $(\phi)$ distribution, chi-square probability $\left(\mathrm{P}\left(>\chi^{2}\right)\right.$ is 0.980 more than 0.05 i.e., 5\% significance level, First order Fourier coefficient $\left(\Delta_{11} / \sigma\left(\Delta_{11}\right)\right)$ to be -2.602 which less than $1.5 \sigma$. The First order Fourier probability $\left(\mathrm{P}\left(>\Delta_{1}\right)\right)$ is found to be 0.021 i.e., $2.1 \%$ less than 0.15 i.e., $15 \%$ significance level, and autocorrelation $(\mathrm{C} / \mathrm{C}(\sigma))$ to be -0.349 less than $1 \sigma$. Here, all these statistical tests supporting isotropy except First order Fourier probability.

The azimuthal angle $(\phi$ - distribution is shown in Fig. 3f, there are several humps at $\left(-25^{\circ}, 35^{\circ}, 45^{\circ}\right)$ and dips at $\left(-45^{\circ}, 5^{\circ}, 25^{\circ}\right)$ are observed. These effects may be due to binning.

\section{CONCLUSION}

This paper studied the spatial orientation of 2,590 galaxies surveyed by SDSS in the Supercluster S $[195+027+0022]$. These data $\left(7^{\text {th }}\right.$ data release $)$ were taken by SDSS Telescope located at Apache Point Observatory, New Mexico, USA, and made available to us by collaboration with the Institute of Astroparticle Physics, Innsbruck University, Austria. From these data, we plotted all-sky diagrams and observed the nature of that plot. We have used the 'PA-inclination method' proposed by 
Flin and Godlowski [10] to convert twodimensional observed parameters to threedimensional galaxy rotation axes (polar and azimuthal angles) and carried out random simulation by generating $10^{7}$ virtual galaxies in order to remove selection effects from the database [11]. To check for isotropy and anisotropy, we have carried out three statistical tests: chi-square, autocorrelation, and the Fourier. There are 'humps' and 'dips' in the polar angle distributions, these 'humps' and 'dips' do not alter the statistics of the sample. Thus we suppose these as binning effects. Similarly in azimuthal distributions, all samples show isotropic distributions as explained above.

Since observed distributions do not vary significantly from the expected distributions for total sample and substructures S1 [195+028+0022], S2 [176+020+0023], and S3 [181+020+0024]. In general, observed that there is no preferred alignment of spin vectors of galaxies. The result of $\theta$-distributions supports the hierarchical clustering scenario (Peebles1969) [3], which predict that the random orientation of the directions of the spin vectors of galaxies. Similarly for the $\phi$-distribution, for total sample and substructures S1 [195+028+0022], S2 [176+020+0023], and S3 [181+020+0024], we found the negative value of first order Fourier coefficient. Therefore in general the spin vector projection of the galaxies tends to oriented perpendicular to the equatorial plane.

\section{ACKNOWLEDGMENTS}

One of the authors (J. R. Malla) wishes to express his gratefulness to the Department of Astro-Particle Physics, Innsbruck University, Austria, for providing data access and also thanks to the authorities of the SDSS for providing the database.

\section{REFERENCES}

[1] Gamow, G. The role of turbulence in the evolution of the universe. Physical Review, 86 (2): 251 (1952).

[2] Von Weizsäcker, C. The evolution of galaxies and stars. The Astrophysical Journal, 114: 165 (1951).

[3] Peebles, P. J. E. Origin of the angular momentum of Galaxies. The Astrophysical Journal, 155: 393402 (1969).

[4] Baier, F. W.; Godlowski, W.; MacGillivray, H. T. Substructures and galaxy orientations in clusters II. Cluster Abell 14. Astronomy and Astrophysics Journal, 403: 847-856 (2003).
[5] Ozernoy, L. The whirl theory of the origin of structure in the universe. In Symposiuminternational astronomical union, 79: 427-438 (1978).

[6] Doroshkevich, A. G. and Shandarin, S. F. A statistical approach to the theory of galaxy formation. Soviet Astronomy, 22: 653-660 (1978).

[7] Aryal, B. \& Saurer, W. Spin vector orientations of galaxies in seven Abell clusters of BM type III. Astronomy and Astrophysics Journal, 432: 841849 (2005).

[8] York, D. G.; Adelman, J.; Anderson, J. E.; Anderson, S. F.; Annis, J.; Bahcall, N. A.; Bakken, J. A. \& 137 more. The sloan digital sky survey: Technical summary. The Astronomical Journal, 120 (3): 1579-1587 (2000).

[9] Holmberg, E. On the apparent diameters and the orientation in space of extragalactic Nebulae. Meddelanden fran Lunds Astronomiska Observatorium Series II, 117: 3-82 (1946).

[10] Flin, P.; Godlowski, W. The Orientation of Galaxy Groups and formation of the local Supercluster, Monthly Notices Royal Astron. Soc. 222 (3): 525-541 (1986).

[11] Aryal, B. \& Saurer, W. Comments on the expected isotropic distribution curves in the galaxy orientation studies. Astronomy and Astrophysics letter, 364: L97-L100 (2000).

[12] Godlowski, W. Galactic orientation within the local Supercluster. Monthly Notices Royal Astron. Soc., 265: 874-880 (1993).

[13] Godlowski, W. Some aspects of the galactic orientation within the local Supercluster. Monthly Notices Royal Astronomy Society, 271: 19-30 (1994). 